\title{
Learning low-dimensional representations via the usage of multiple-class labels
}

\author{
Nathan Intrator $†$ and Shimon Edelman $\ddagger \|$ \\ $\dagger$ Institute for Brain and Neural Systems, Brown University, Providence, RI 02192, USA \\ $\ddagger$ Center for Biological and Computational Learning, MIT E25-201, Massachusetts Institute of \\ Technology, Cambridge, MA 02142, USA
}

Received 18 February 1997

\begin{abstract}
Learning to recognize visual objects from examples requires the ability to find meaningful patterns in spaces of very high dimensionality. We present a method for dimensionality reduction which effectively biases the learning system by combining multiple constraints via the use of class labels. The use of extensive class labels steers the resulting lowdimensional representation to become invariant to those directions of variation in the input space that are irrelevant to classification; this is done merely by making class labels independent of these directions. We also show that prior knowledge of the proper dimensionality of the target representation can be imposed by training a multi-layer bottleneck network. Computational experiments involving non-trivial categorization of parameterized fractal images and of human faces indicate that the low-dimensional representation extracted by our method leads to improved generalization in the learned tasks and is likely to preserve the topology of the original space.
\end{abstract}

\section{Introduction}

Learning to recognize visual objects in images-represented as collections of pixel valuesrequires the ability to find meaningful patterns in spaces of tens or hundreds of thousands of dimensions. The resulting need for models with an extremely large number of parameters raises the problem of sparse data: the number of observed samples (training patterns) is smaller than or similar to the number of model parameters that must be estimated. Even simple and widely used methods, such as linear discriminant analysis (Fisher 1936), should be adjusted to reflect the low ratio of training samples to the number of parameters (Buckheit and Donoho 1995). Thus, it is clear that neural network learning methods require special care when applied to problems arising in vision.

\subsection{General approaches to the facilitation of learning}

A fundamental assumption frequently made in an attempt to alleviate the problem of sparse data, also known as the curse of dimensionality (Bellman 1961), is the existence of a lowdimensional representation (LDR) of the problem space. Yet, postulating that an LDR exists does not provide an efficient way to find it. To do so, one may, for instance, further assume that the data are clustered. If data points belonging to the same class are indeed clustered

$\S$ E-mail: nin@cns.brown.edu. On leave from School of Mathematical Sciences, Tel-Aviv University, Israel.

|| E-mail: edelman@ai.mit.edu. 
in the high-dimensional space, a useful LDR can be found by looking for projections that emphasize the cluster structure (Intrator 1993).

More generally, innovative use of the training data is needed. For example, methods for data reuse, such as cross-validation (Stone 1974) and bootstrap (Efron and Tibshirani 1993), can help in obtaining confidence intervals (Baxt and White 1995) and improved performance (Breiman 1992, Breiman 1994, LeBlanc and Tibshirani 1994) of learning networks. Smooth bootstrap (Efron and Tibshirani 1993) can also increase the independence among predictors for the purpose of ensemble averaging (Raviv and Intrator 1996). Such methods lead to a reduction in the variance portion of the error, with little or no effect on the bias of the predictor.

One can control the variance portion of the error also by imposing global assumptions about the nature of the predictor that is to be learned. These include smoothness (Wahba 1990, Poggio and Girosi 1990) as well as assumptions about the distribution of the parameters, e.g. favouring small weights via a weight decay process or favouring particular distributions such as mixtures of Gaussians (Nowlan and Hinton 1992). A general framework for imposing such constraints is presented by Intrator (1993).

\subsection{Specific assumptions and prior knowledge}

Unlike data, class labels are not often reused to facilitate learning (see, however, Grossman and Lapedes (1993)). In particular, few learning algorithms can accommodate multiple-class labels, which are likely to contain useful information regarding the structure of the data. Furthermore, humans make natural use of the knowledge that objects may have several class associations (say, at different category levels). In contrast, in machine learning, it is not clear how one should proceed given multiple-class or hierarchical labels, and whether such information can be used effectively or at all. We believe that through the use of multiple-class associations, learning can be constrained (biased) towards a better solution, and that innovative use of multiple-class labels may be a practical way to introduce prior knowledge into a high-capacity learning machine. We present a method for introducing such prior information during training, while avoiding the need to construct different lowlevel representations for different tasks defined on the same data. This approach naturally facilitates generalization across tasks, also known as transfer of skill-a hallmark of human cognitive prowess (see section 1.1 of Intrator and Edelman (1996) for a review). It has been observed in the past that training a classifier on multiple tasks (using the same data) may be an efficient way to introduce desirable bias into the solution (Caruana 1993) and to improve generalization (Caruana 1995). We further argue that forcing the learning system to use multiple-class label information, and letting it ignore dimensions of data variation with respect to which invariance is required, leads to the extraction of an LDR that captures the dimensions relevant to the task, and is orthogonal to the dimensions along which the variance is irrelevant (e.g. the orientation of an object in a visual recognition task). Note that a complementary approach is to learn, instead of a variety of labelling schemes for a given data set, the transformations which leave its members invariant (Lando and Edelman 1995) or the invariances of the individual data items (Simard et al 1992, Thrun and Mitchell 1995).

\subsection{Topology-preserving dimensionality reduction}

As we shall see, generating an LDR through the use of multiple-class labels generally results in the preservation of the topology of a low-dimensional space containing the examples (section 4). As topology-preserving mapping is the ultimate goal of a number of methods of dimensionality reduction, it is appropriate to mention here the typical approaches taken 
by these methods. The oldest among these is multidimensional scaling (MDS) (Young and Householder 1938) which is discussed in the appendix. The main problem with MDS, if it is considered as a method for massive dimensionality reduction rather than as a tool for the exploration of experimental data in applied sciences (Shepard 1980, Siedlecki et al 1988), is its poor scaling with dimensionality.

In the context of learning, a number of methods for topology-preserving dimensionality reduction have been derived from the idea of a self-supervised auto-associative network (Elman and Zipser 1988, DeMers and Cottrell 1993, Leen and Kambhatla 1994, Demartines and Hérault 1996). Because these methods are unsupervised, they extract representations that are not necessarily orthogonal to the irrelevant dimensions of the input space. An interesting approach that combines supervised feature extraction with topology preservation was proposed by Koontz and Fukunaga (1972) and Webb (1995), whose dimensionality reduction algorithms explicitly optimize a joint measure of class separation and (inputspace) distance preservation. This approach, which resembles MDS, suffers from the same poor scaling with the dimensionality. The performance of a radial basis function network as a topology-preserving dimensionality reduction method has been studied by Lowe and colleagues (Lowe 1993, Lowe and Tipping 1996) and compared with other methods including MDS and Kohonen and Sammon mapping.

\subsection{Overview of the paper}

In the present paper, we use objects belonging to parametrically defined low-dimensional families to demonstrate that training with a combined objective of (1) discrimination among labelled categories known to reside within the same domain and (2) explicit collapse of dimensions over which discrimination is to be generalized, leads to a reliable recovery of the target low-dimensional manifold. Our method is effective even when the manifold to be extracted from the data is curved (i.e. when the problem is nonlinear), and is embedded in a measurement space of nearly a thousand dimensions. Furthermore, it allows the construction of classifiers of considerably lower complexity for other tasks involving the same objects, compared to what is possible under the usual approach of learning a separate representation for each task. As a control, we show that neither principal component analysis of the data, nor its nonlinear extension (implemented, respectively, by three-layer and by five-layer 'bottleneck' autoencoder networks) can extract a meaningful LDR from our data. In addition we show that if class labels are not used to specify the invariance dimensions (i.e. directions that are orthogonal to the target LDR manifold), the extraction of the LDR also fails.

The paper is structured as follows. Section 2 introduces the fractal patterns and the face images, and explains the manner in which these data sets were generated to facilitate subsequent evaluation of the LDR extraction method. Section 3 explains in detail the variations on the basic LDR extraction method that we devised. Section 4 then presents the results obtained from a computational evaluation of the different methods. Finally, section 6 summarizes the results and relates them to some recent evidence of the relevance of dimensionality reduction to biological vision.

\section{Data sets}

To test the ability of a neural network to discover simple structure embedded in a highdimensional measurement space, we created two data sets, in both of which the discovery of the LDR requires a highly nonlinear transformation on the measurement space. In the creation of each data set, we started with a two-dimensional parametric representation space, 
in which we placed 18 classes of objects on a regular $3 \times 6$ grid; an additional parametric dimension, orthogonal to the first two, modelled the within-class variation (see figure 1). The first data set, FRACTALS, was computer-generated, while the second set, FACES, was derived from natural visual stimuli (3D laser-scanned human heads).

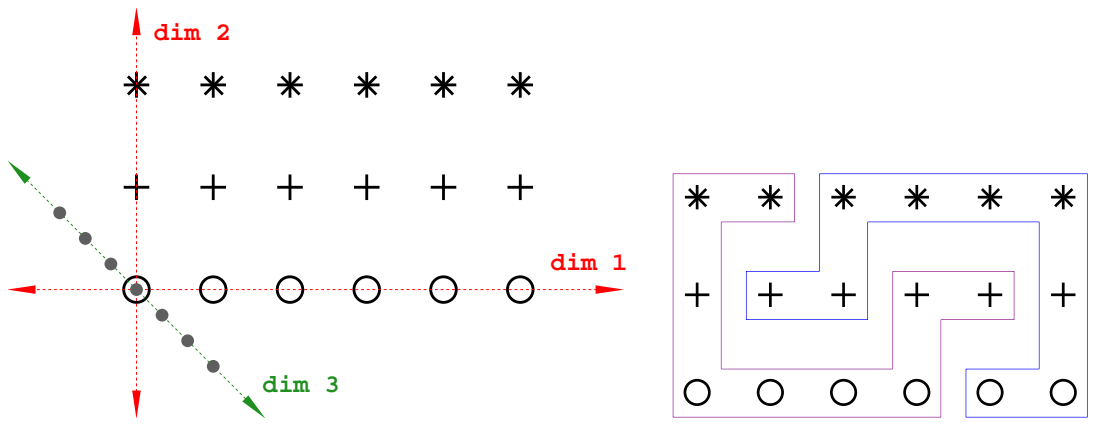

Figure 1. Left: the parametric representation from which the high-dimensional data sets were created. dim 3 is the within-class dimension of variation, used in training the LDR extractors, as explained in section 3 (see also figure 6). Right: the dichotomy classification task, used in testing the LDR.

\subsection{Fractals}

The patterns in the FRACTALS data set were generated using publicly available software (Xfractint 2.03). We chose the quaternion Julia set (entry quatjul in the Xfractint pattern menu), which is parametrized by six variables and therefore can be used for generating complicated patterns that depend on up to six parameters. The quatjul iteration formula is

$$
\begin{aligned}
& q(0)=\left(\text { xpixel, ypixel }, z_{j}, z_{k}\right) \\
& q(n+1)=q(n) * q(n)+c
\end{aligned}
$$

where both $q$ and $c=\left(c_{1}, c_{i}, c_{j}, c_{k}\right)$ are quaternions (for further details, see Pickover (1990), chapter 10). The three dimensions shown in figure 2 correspond to the variation of parameters $c_{1}, c_{j}$, and $c_{k}$, respectively.

Note that the transformation from the 3D parameter space to the image space, implied by the above formula, is highly nonlinear. To quantify this characteristic of the data set, which bodes severe problems for linear projection methods for dimensionality reduction, we have carried out a principal component analysis (PCA) of the data. We found that projection on the first 2, 5, and 13 eigenvectors accounted for $16.8 \%, 31.8 \%$, and $58.6 \%$ of the variance, respectively (see figure 3 (top); the dimensionalities specified above will feature in the reports of the performance of the different LDR extraction methods, listed in section 4).

To what extent could the PCA-derived LDR be regarded as a good replica of the original 2D parametric space in which the data were embedded? We tested the 13-dimensional PCA-derived projection of the data for planarity using multidimensional scaling (MDS) - a procedure designed to embed data points into a metric space of specified dimensionality (in this case, 2D), while preserving as much as possible the distances between the points (see the appendix for details and references on MDS). We found that the residual stress resulting from embedding the 13-dimensional PCA subspace into 2D was 0.25 (the stress vanishes for point configurations that can be embedded into the target space without distortion, and 

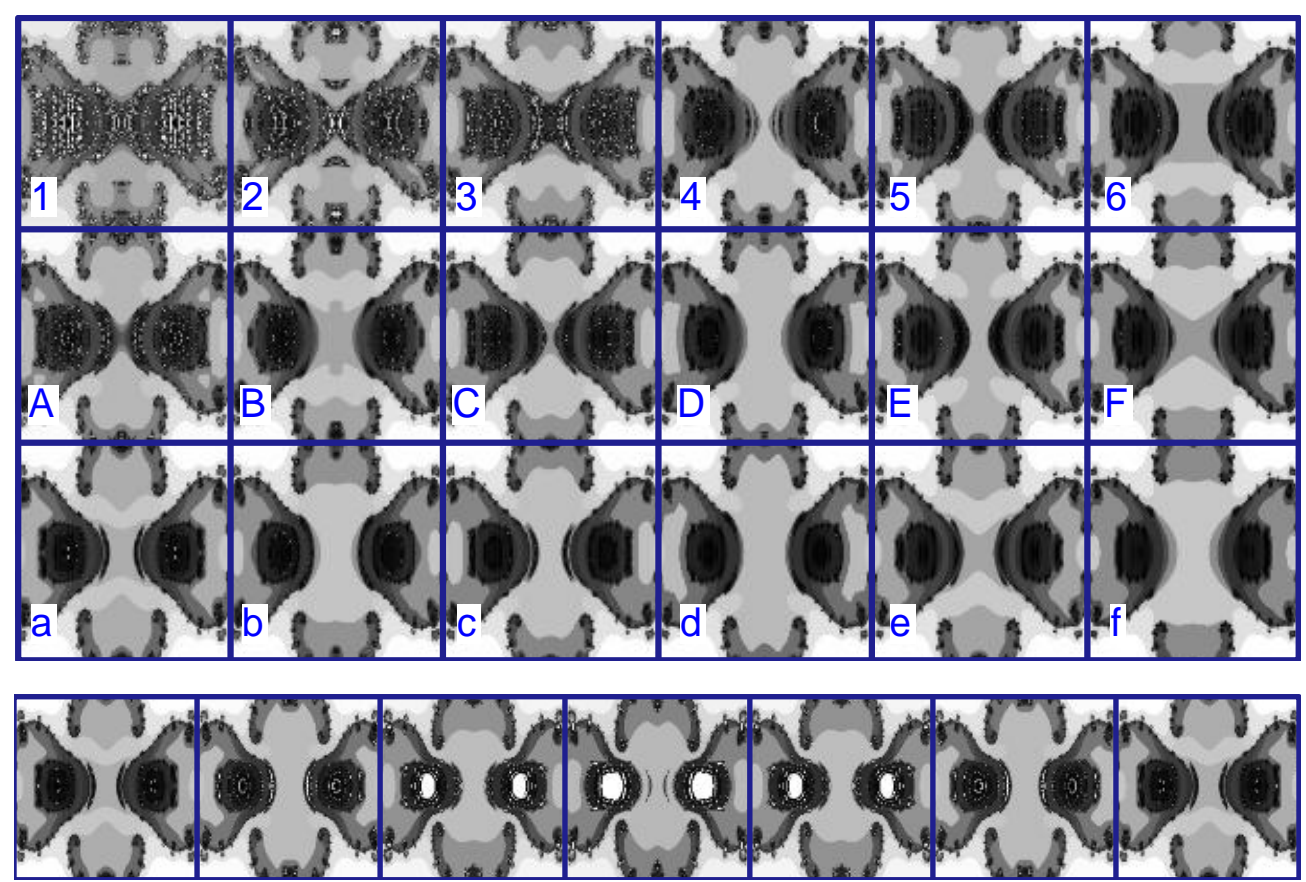

Figure 2. Some of the images from the FRACTALS data set (section 2.1). The 18 images in the upper part of the figure correspond to the 18 classes of fractal objects; only one member of each class, corresponding to one value along the third dimension of variation, is shown (cf figure 1). The seven images in the lower part of the figure are the seven exemplars from class a. Prior to classification, the images, originally of size $256 \times 256$, were reduced to $28 \times 28=784$ dimensions by correlation with a bank of filters (section 2.3).

approaches unity for poor embeddings). Furthermore, the 2D configuration of the data points derived by MDS from the inter-point distances in the 13 PCA dimensions (when these were forced into a $2 \mathrm{D}$ space) bore no resemblance to the $3 \times 6$ parametric grid pattern built into the stimuli. Thus, we conclude that the LDR extraction problem in the present case is indeed highly nonlinear.

\subsection{Faces}

We chose human faces as the basis for our second data set, mainly to facilitate intuitive understanding of the computational experiments and their expected results. Of all natural objects, faces are the category with which human observers are the most proficient; we surmised that the orthogonal manipulations involved in the generation of the two betweenclasses and one within-class dimensions of image variations would be most easily perceived if the images were those of faces. Since face images possess no inherent low-dimensional parameterization (unlike fractal patterns generated by a known algorithm) we had to impose such a parametrization on the data. We chose to do it by starting with a set of nine 3D laser scans of human heads (see figure 4), and by embedding the $3 \times 6$ grid in the 2D space spanned by the two leading 'eigenheads' obtained from the data by PCA $\dagger$.

$\dagger$ A similar approach to the generation of parametrically controlled head stimuli has recently been proposed by Atick et al (1996). 

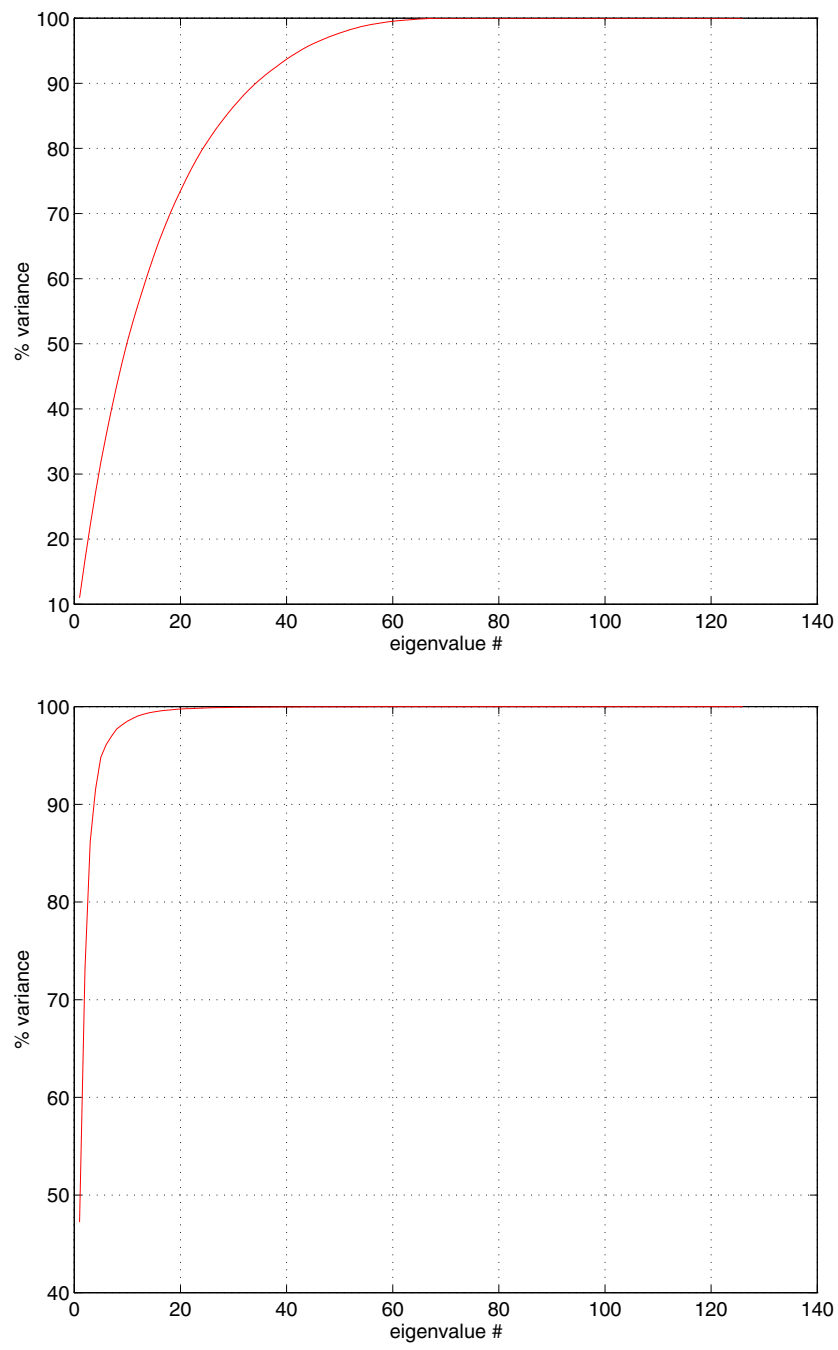

Figure 3. Cumulative percentage of variance plotted vs. the number of participating eigenvector projections, as obtained by principal component analysis (PCA) on the FRACTALS data set (top) and of the FACES data set (bottom).

Each of the 18 heads derived by PCA from the original scanned head data was piped through a graphics program, which rendered the head from seven viewpoints, obtained by stepping the (simulated) camera in $3^{\circ}$ rotation steps around the midsagittal axis. The rendering program assumed a semi-glossy reflectance model for the head surface; because of that, and because of the trigonometric functions involved in the viewpoint transformation, we expected a nonlinear relationship between the planar 2D configuration formed by the 18 heads in the parameter space and the space spanned by the rendered images of these heads. As with the FRACTALS data set, we quantified the degree of nonlinearity by subjecting the image data to PCA and to MDS analysis. For the FACES data set, the nonlinearity was somewhat more moderate than for FRACTALS, but still considerable: projection onto the first two and the first 13 eigenfaces accounted for $73.1 \%$ and $99.2 \%$ of the variance, respectively; 


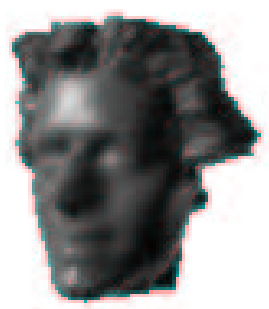

D

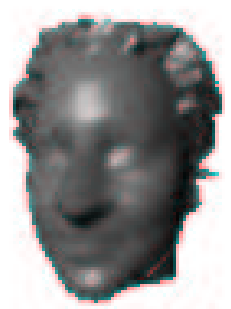

$\mathbf{J} \mathbf{i}$

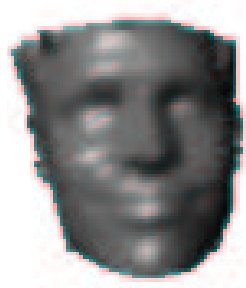

$\mathbf{P}$

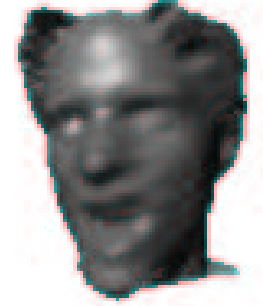

$\mathrm{Ha}$

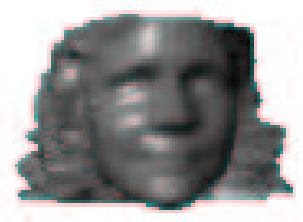

Jo

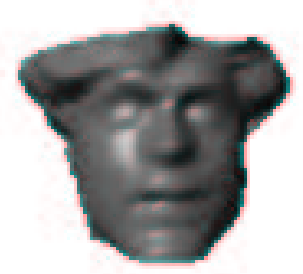

$\mathbf{R}$

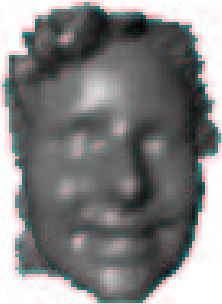

$\mathrm{He}$

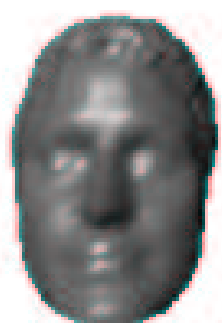

$\mathbf{K}$

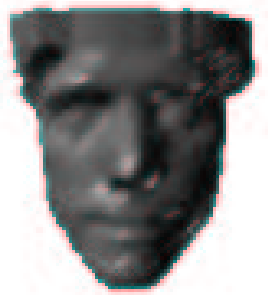

S

Figure 4. The nine 3D laser scans of human heads used to generate the face images shown in figure 5. Three are distributed with Silicon Graphics Inc. systems, and the other six are available via anonymous ftp, courtesy of Cyberware Inc., as part of their demonstration software.

the subspace spanned by the first 13 eigenfaces was still significantly non-planar, as indicated by the residual stress of MDS, which was equal to 0.16 .

\subsection{Preprocessing}

The images generated by the two methods described above were imported into Matlab ${ }^{\mathrm{TM}}$, and were preprocessed prior to LDR extraction. The FRACTALS data were subjected to histogram equalization, then convolved with a bank of $28 \times 28=784$ receptive fields (Matlab Image Processing toolbox; Laplacian of Gaussian, kernel size 9, $\sigma=0.6$ ). Images in the FACES data set, originally of size $400 \times 400$, were reduced to $49 \times 16$ dimensions by cropping the background and by correlation with a bank of filters (Matlab Image Processing toolbox; Laplacian of Gaussian, kernel size 11, $\sigma=0.9$ ). In both cases, the preprocessing reduced the dimensionality of the data from 65536 to 784 , and served as a crude approximation of the transformations that a stimulus undergoes on its way to the primary cortical visual area in the mammalian brain. 

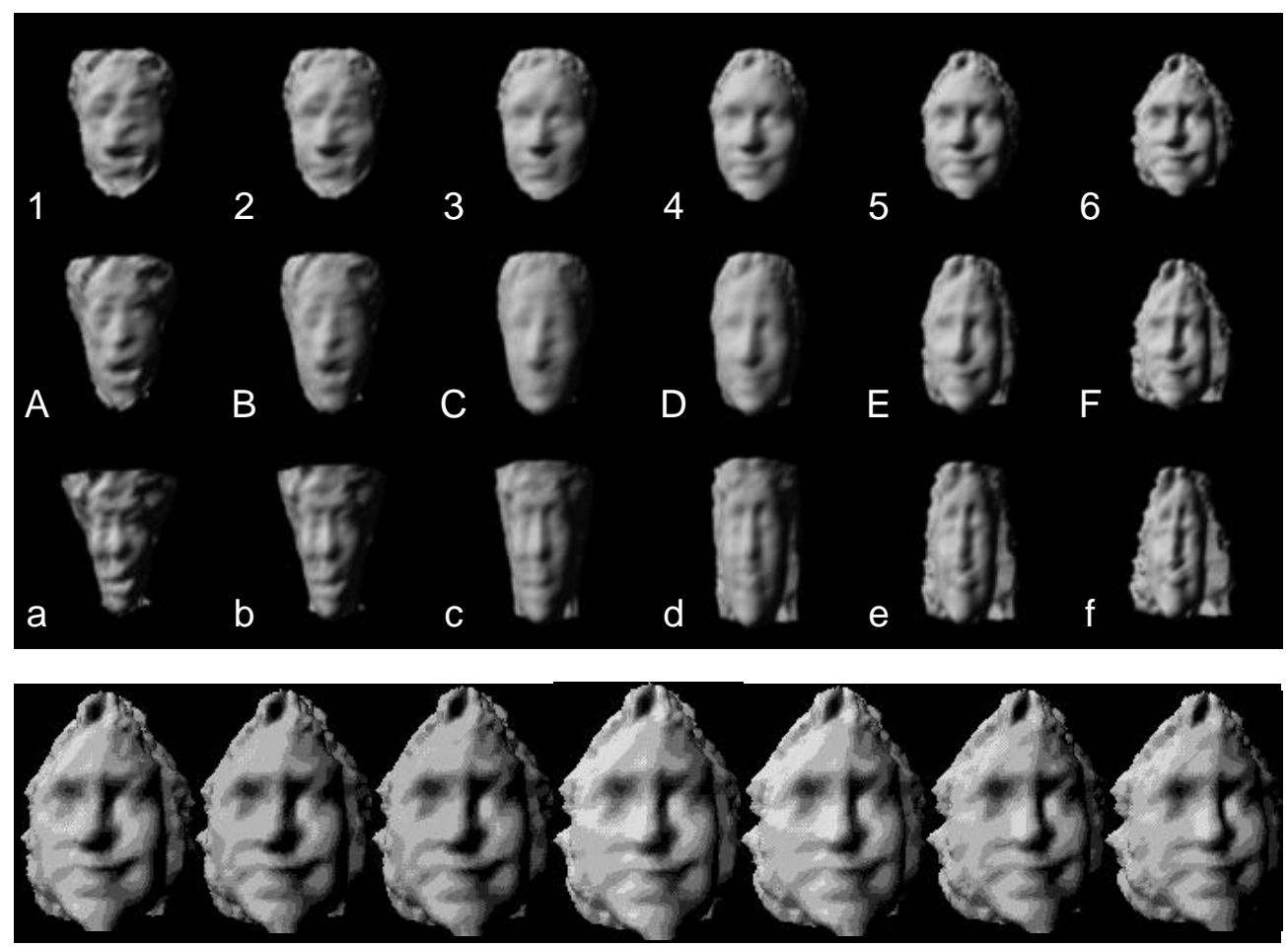

Figure 5. Some of the images from the FACES data set (see section 2.2). Top: the 18 heads obtained by placing a $3 \times 6$ grid in the space of the two leading principal components of the original nine heads. Bottom: the seven views of the rightmost head in the top row above; the views differ by $3^{\circ}$ steps of rotation in depth, summing up to a total difference of $18^{\circ}$. It should be noted that orientation differences of up to $20^{\circ}$ go unnoticed by human viewers (Busey et al 1990), while presenting non-trivial problems for neural networks, which must be trained explicitly to compensate for or to tolerate the misorientation. Prior to classification, the images, originally of size $400 \times 400$, were reduced to 784 dimensions by cropping the background and by correlation with a $49 \times 16$ bank of Gaussian-profile filters (section 2.3).

\section{Methods}

All our computational experiments consisted of two phases: training the LDR extractor, and evaluating its performance (see figure 6). We now describe these two procedures in detail (the results of the experiments are reported in section 4).

\subsection{Derivation of the $L D R$}

As we argued in the introduction, a faithful low-dimensional representation of the data space should serve as a good basis for efficient classification of the data; the aim of the experiments we describe here was to determine whether a classifier, faced solely with the task of learning to label a selection of data points, is likely to do so by deriving an LDR that would help it in the classification process. Note that the extraction of an LDR was made possible, at least in principle, by the design of the data sets, into which we built a low-dimensional structure. At the same time, the embedding of this structure in a high-dimensional image 


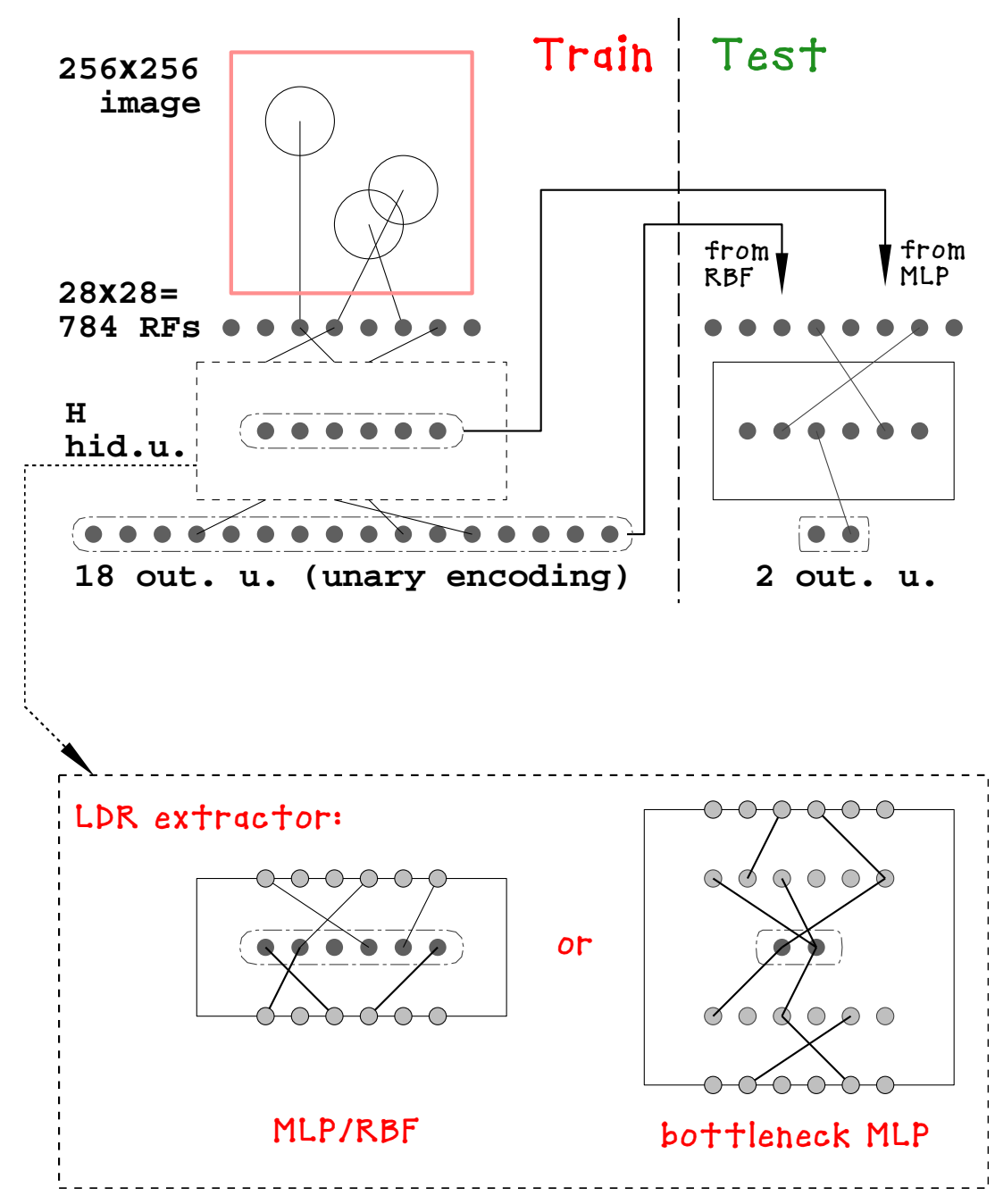

Figure 6. The low-dimensional representation (LDR) extraction scheme (see section 3). The LDR extraction network appears on the left, under the label Train. Following preprocessing (see section 2.3), a learning module - a multi-layer (here, 3-layer) perceptron (MLP), a 5-layer 'bottleneck' MLP or a radial basis function interpolator (RBF) - is trained to produce a unary encoding of the class labels associated with the input image (i.e. an 18-dimensional vector in which the value of only one dimension is nonzero for any given input). The testing procedure (illustrated on the right, under the label Test) is described in detail in the text.

space, which, moreover, was nonlinearly related to the original parametric representation of the data, made the extraction of LDR a highly non-trivial task.

3.1.1. One hidden layer (1-HL) MLP classifier. Researchers have explored in the past the ability of multilayer perceptrons, trained as autoencoders, to form low-dimensional representations of the data in the hidden layer (DeMers and Cottrell 1993). In the autoencoder approach, the data are forced to pass through a low-dimensional 'bottleneck'the hidden layer of an MLP. The network is taught to reconstruct the input patterns from the LDR formed at the hidden layer. A major disadvantage of this approach is that it is 
inherently oblivious to any regularities that may be present in the data†. For example, face images depend not only on the identity of the person, but also (sometimes in an overwhelming fashion) on the viewing conditions; a resourceful LDR extractor attempting to recover a low-dimensional 'face space' from a batch of face images should take this into account and discount the 'irrelevant' dimensions (in this example, variations due to viewpoint and illumination), while preserving the relevant ones.

One way to bias the LDR extractor to do that is by training it as a classifier, rather than as an autoencoder, and, in particular, to introduce dimensions to which the LDR should be orthogonal. Specifically, we propose to look for the LDR in the hidden layer of an MLP trained to classify the input images into a relatively large number of classes (striving for adequate coverage of the postulated low-dimensional pattern space), while ignoring withinclass variation due to extraneous factors. In the present case, the data sets were broken into 18 distinct classes, placed on a grid in two parametric dimensions; the exemplars within each class differed along a third dimension, which the MLP had to learn to ignore (in the FACES data set, this was the face orientation dimension).

3.1.2. Three hidden layer (3-HL) bottleneck MLP classifier. Because the regular three-layer (1-HL) MLP, if trained as an autoencoder, is known to carry out an approximate PCA of the data (Cottrell et al 1987, Baldi and Hornik 1989, Oja 1989), we could not expect it to perform too well on our nonlinear data sets. A natural modification of the 1-HL MLP architecture, which may be better suited to nonlinear LDR extraction, is the three hidden layer (3-HL) MLP (Leen and Kambhatla 1994). We have, therefore, chosen to explore this approach, but, following the considerations stated above, the 3-HL MLP was to be trained as a classifier, and not as an autoencoder.

The results obtained from the 3-HL experiments further indicate that it is the specific training task (which included a specific generalization subtask) that led to a useful LDR, while a similar and even more powerful architecture without the generalization task was unable to recover a useful LDR.

3.1.3. RBF classifiers. The third LDR extraction method that we have chosen to examine is projection into a space spanned by the responses of a number of 'prototype detectors' (Edelman 1995b, Edelman et al 1996). Each detector, which can be realized as a radial basis function (RBF) classifier, is trained to output a constant value for a set of instances of a given class (e.g. images of a given face taken from a series of viewpoints). If the RBF module successfully generalizes to other instances of the same class by maintaining a relatively unperturbed response level for those inputs, and if its response drops off monotonically with increasing distance between the input and the optimal stimulus (the prototype for that module), a collection of modules tuned to different classes forms a distributed representation of the input that is likely to capture the low-dimensional structure of the input space (Edelman and Duvdevani-Bar 1997b).

For the present purpose, we trained a single RBF network to output a unary representation of the class membership, as we did with the MLP classifiers described above (this is equivalent, of course, to training 18 separate RBFs, sharing the same 'hidden' or basis function layer). Training was confined to the computation of the optimal hidden to output weights (the basis functions being centred on a subset of the input examples), and could be carried out, therefore, by simple pseudoinverse (Poggio and Girosi 1990). As a 
result, this LDR extraction method was much faster than the two methods involving MLPs (which were trained by back-propagation). As we shall see, this rapid LDR extraction produced representations nearly as good as those obtained by MLP.

\subsection{Evaluation of the $L D R$}

In the second training phase (as seen in figure 6) we used only LDRs which had been successful at the first stage in the multiple-label classification task involving the training set. Specifically, after training for a fixed number of epochs, if classification performance (on the training data set), was above $80 \%$ or $90 \%$ (depending on the difficulty of the task), the LDR was approved for use at the second stage, involving the two-class dichotomy. If the performance in a given trial was satisfactory according to this criterion, the LDR was evaluated using three different methods, as described below. Note that the data at this point consisted of 54 vectors (18 classes times 3 exemplars), of dimensionality that depended on the LDR extraction method, and varied between 2 (for the 3-HL bottleneck MLP classifier) and 13 (for the RBF classifier).

3.2.1. RBF performance in the dichotomy task. The first method was designed to assess the utility of the LDR for supporting a representative classification task not directly related to the one-out-of-18 classification used to train the LDR extractor. For that purpose, the recovered LDR was used to train an RBF classifier on a nonlinear two-class (dichotomy) problem (see figure 1, right). The generalization performance of this classifier was then compared with that of an identical classifier trained on the raw 784-dimensional filter-space representation of the image set, on the same dichotomy problem.

3.2.2. MDS. The second method was designed to allow visualization of the configuration formed by the 18 classes in the LDR space. Because in general this space had more than two dimensions (except in the case of the 3-HL bottleneck MLP), the points had to be embedded in two dimensions for easy plotting and visualization. This embedding was carried out by multidimensional scaling (MDS), which accepted a $54 \times 54$ table of mean inter-point distances and produced an embedding of the 54 points (each corresponding to a single test instance) into two dimensions. The mean-distance table was computed by element-wise averaging of a number of tables arising from repeated trials; the LDR extraction experiment was run repeatedly to reduce the dependence of the results on the randomized initial conditions, to which MLP training is known to be sensitive.

3.2.3. 3-HL bottleneck MLP. As mentioned above, the LDR derived by the 3-HL bottleneck MLP with two units in the middle hidden layer is two-dimensional, and thus can be visualized directly, without the mediation of MDS (cf. figure 8). Note that in this case the results of repeated runs cannot be simply averaged (unless they are first converted into the distance-table format, which would necessitate subsequent application of MDS). To combine the results of a number of runs we used Procrustes transformations (Borg and Lingoes 1987) to normalize (scale, rotate and translate) all the LDR configurations to the configuration obtained in the first run $\dagger$. As a direct control of the outcome of MDS-based visualization, we also ran the 3-HL bottleneck MLP on the LDRs obtained by other methods,

$\dagger$ Procrustes, or similarity, transformations, by definition, leave the shape of a set of points unchanged—a property that is useful for comparing a number of configurations that have similar shapes, but may differ in size, orientation, and location relative to a coordinate system. 
as a kind of post-processing. In the next session, the LDR configurations derived by the different methods are plotted alongside each other, where appropriate.

\section{Results}

We now proceed to describe the results of the computational experiments in dimensionality reduction that we performed on the FRACTALS and the FACES data sets. The same experiments were carried out in both cases; the reported results include:

(i) evaluation of the three methods of LDR extraction listed in section 3.1;

(ii) a control comparison between the LDR obtained with 18-class training with that obtained with two-class training;

(iii) A plot of the 2D configuration derived from the raw 784-dimensional data by MDS.

We also performed some additional experiments with the FRACTALS data set, which explored the effect of imposing categorical structure on the LDR and are reported in the next section. Information regarding other experiments with the FRACTALS data (namely, a study of the effect of the number of bases on the quality of the LDR extracted by an RBF network, and a comparison of LDRs derived from several versions of FRACTALS data of varying difficulty) can be found in Intrator and Edelman (1996).

\subsection{The FRACTALS data set}

The LDR configuration obtained on the FRACTALS data by the 1-HL MLP method is shown in figure 7 (left). The first striking feature of this configuration is its mandala-like general structure: the MLP network did a good job of spreading the 18 classes as far apart as possible from each other, while preserving the grouping of the triplets of points corresponding to different exemplars of the same class. The second notable feature emerges from a scrutiny of the relative locations of the classes belonging to the same six-class row (see figure 1, left). Each of the three rows is marked by a different symbol $(\circ,+$, and $*)$; the six clusters in a row are labelled consecutively (1-6, A-F, a-f). Note that each row curves upon itself; e.g. clusters 2, 3 and 4 are progressively farther away from cluster 1, while clusters 5 and 6 are progressively closer to cluster 1 . An intuitive explanation of this pattern may lie in the non-monotonic (cyclical) dependence of the appearance of fractal images produced by the quatjul procedure under progressively larger parametric change on the parameter-space distance to some reference image.

Figure 7 (right) shows the configuration derived by a 1-HL MLP trained on a dichotomy. Even though the resulting LDR is about as good for supporting this dichotomy as the one obtained following 18-class training, it is much worse as far as the faithful representation of the original parameter space is concerned: the 18-cluster structure is lost in this LDR.

The performance of the 3-HL bottleneck MLP in LDR recovery is illustrated in figure 9. Note that in this case the LDR can be read off the middle hidden layer of the MLP (which contained two hidden units) and plotted directly, without post-processing by multidimensional scaling.

The recovery of LDR by an RBF classifier (the third method we explored) is illustrated in figure 10. Note that the mandala-like structure in this plot prevails over the preservation of the within-row order of clusters (cf figure 7). Thus, the representations derived by MLPbased methods are more faithful than those obtained by RBFs to the true low-dimensional parametric variation built into the data. 

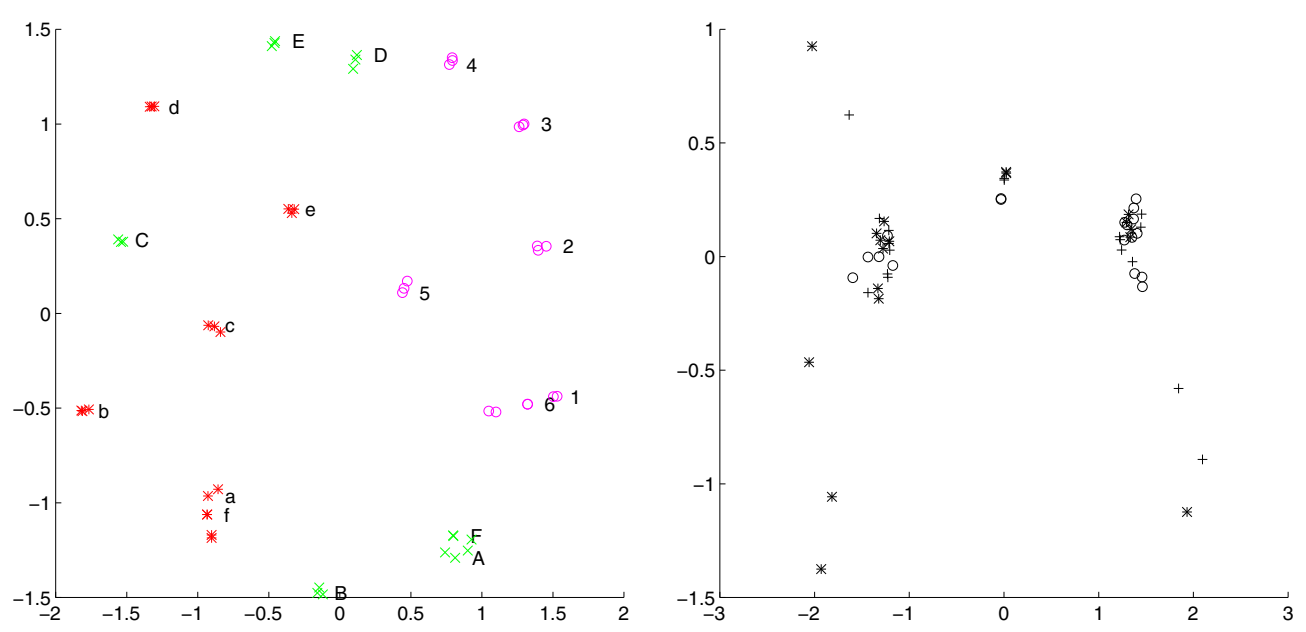

Figure 7. FRACTALS data, LDR by 1-HL MLP with five hidden units (section 3.1.1), MDS visualization (section 3.2.2). Left: MLP trained on 18 classes; here and in the subsequent plots, the three different symbols $(\mathrm{o},+$ and $*)$ correspond to points belonging to the different rows of figure 1. Note the good separation of the 54 points into 18 classes; the three points in each class (corresponding to the three test images per class) are usually clustered together. Right: MLP trained on a dichotomy; two rather than 18 clusters are apparent. The test dichotomy classification error (section 3.2.1) was typically about 0.05 in both cases, compared to about 0.3 on the raw data.
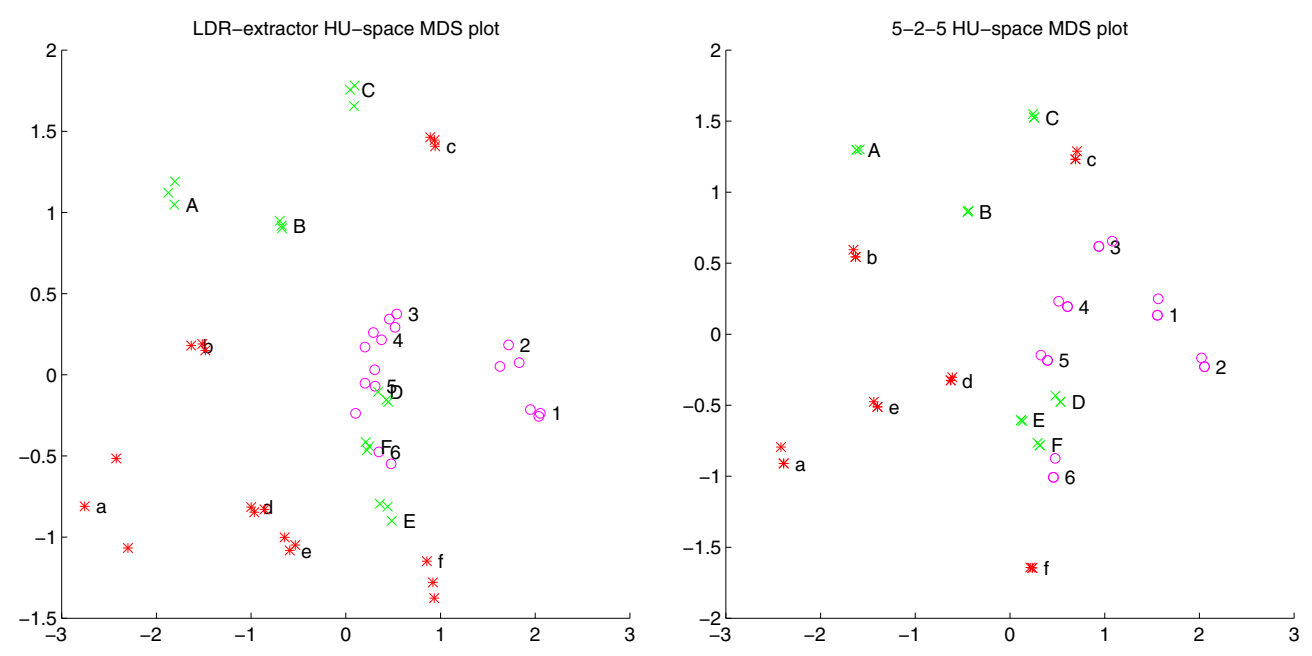

Figure 8. FRACTALS data, LDR by 1-HL MLP with five hidden units. Left: MDS visualization. Right: 3-HL bottleneck MLP visualization. The two visualization methods yield similar configurations, although they rely on entirely different embedding algorithms. This adds credibility to the use of distance (or distance rank) preserving methods for embedding data in points in two dimensions for the purpose of visualization (cf Sammon (1969) and Siedlecki et al (1988)).

We next illustrate the ability of the MLP-based LDR extraction method to assimilate hierarchical category knowledge in a natural manner. In the first experiment that examined 

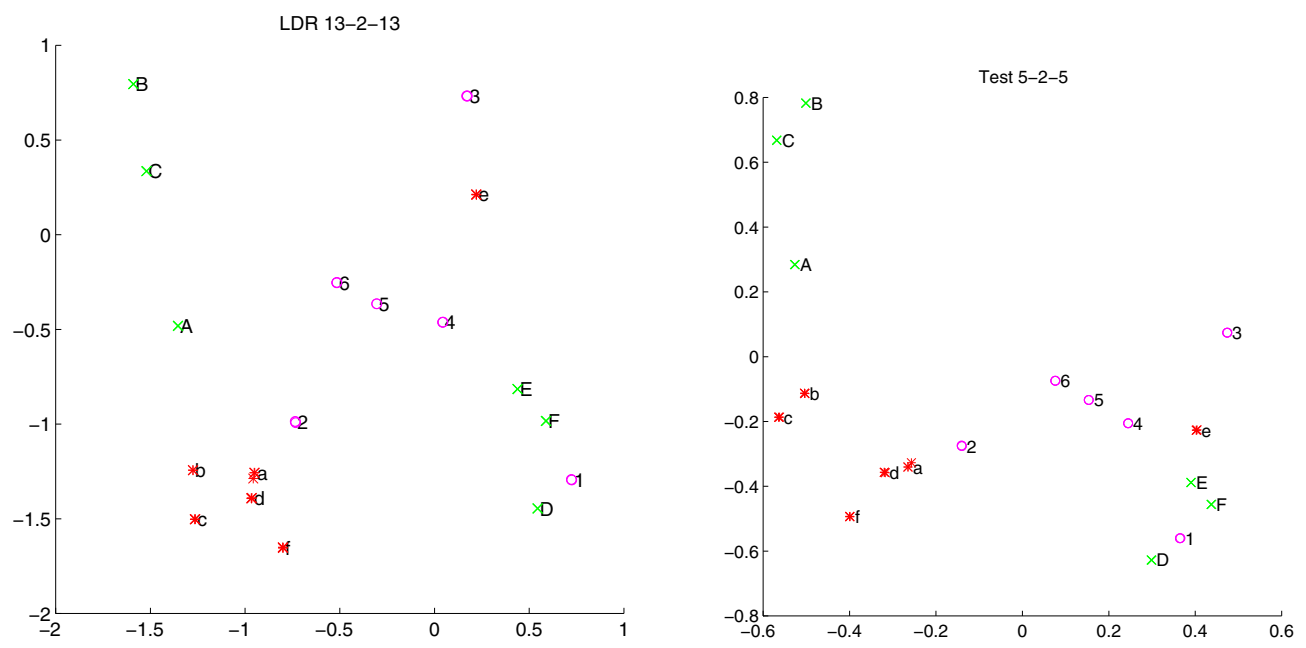

Figure 9. FRACTALS data, LDR by 3-HL bottleneck MLP (section 3.1.2), Procrustes visualization (section 3.2.3). Left: results for 3-HL bottleneck MLP with two hidden units, trained with class labels on the filter data. The test dichotomy error rate was 0.03 , compared to 0.41 on the raw data. Right: results for 3-HL bottleneck MLP, trained as an autoencoder on the LDR derived from the middle HL of the previous 3-HL MLP.
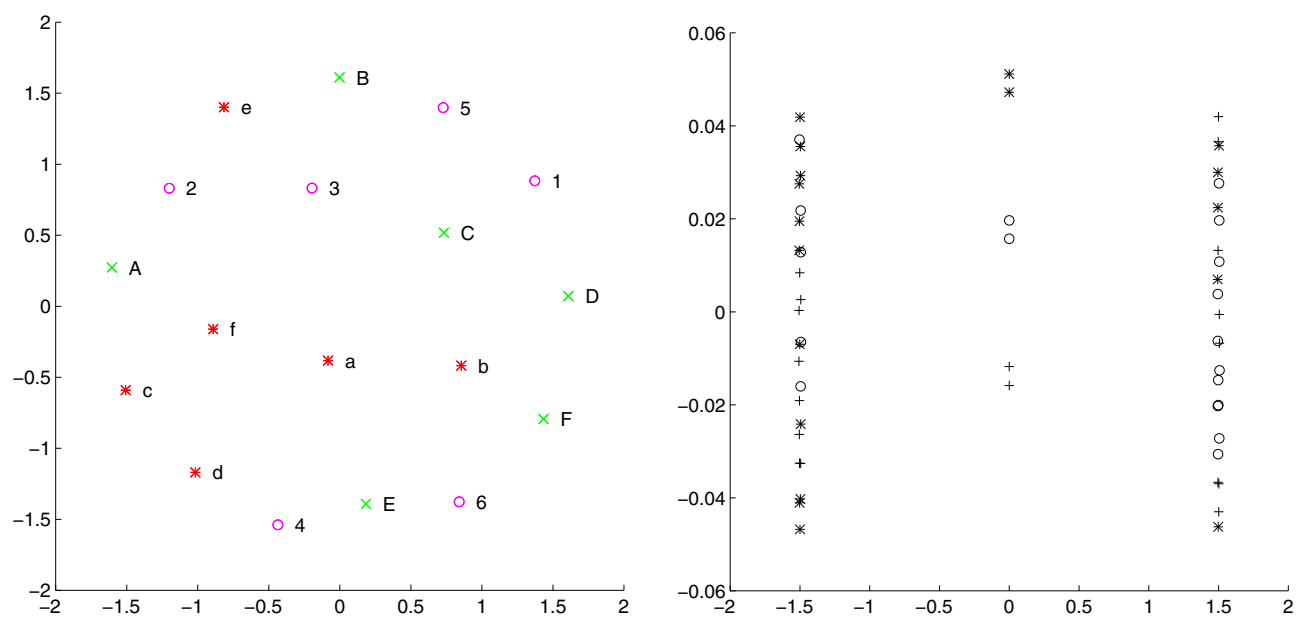

Figure 10. FRACTALS data, LDR by RBF (section 3.1.3), MDS visualization. Left: results for a 72-centre RBF; test dichotomy error 0.0 , compared to 0.24 on the raw data; Right: control results obtained by a 72-centre RBF trained on a dichotomy; test dichotomy errors as above. A parametric study of the performance of this method on the number of centres of the RBF network can be found in Intrator and Edelman (1996).

this issue, three higher-level class labels were added to the set of 18 labels normally used in the training stage. For each data point, the higher-level label indicated the row to which it belonged (see figure 1). In the resulting configuration, the 18 clusters were separated, on a coarser level, into three groups, corresponding to the three higher-level class labels (see figure 11). In the second experiment, the LDR extractor was taught three labels 

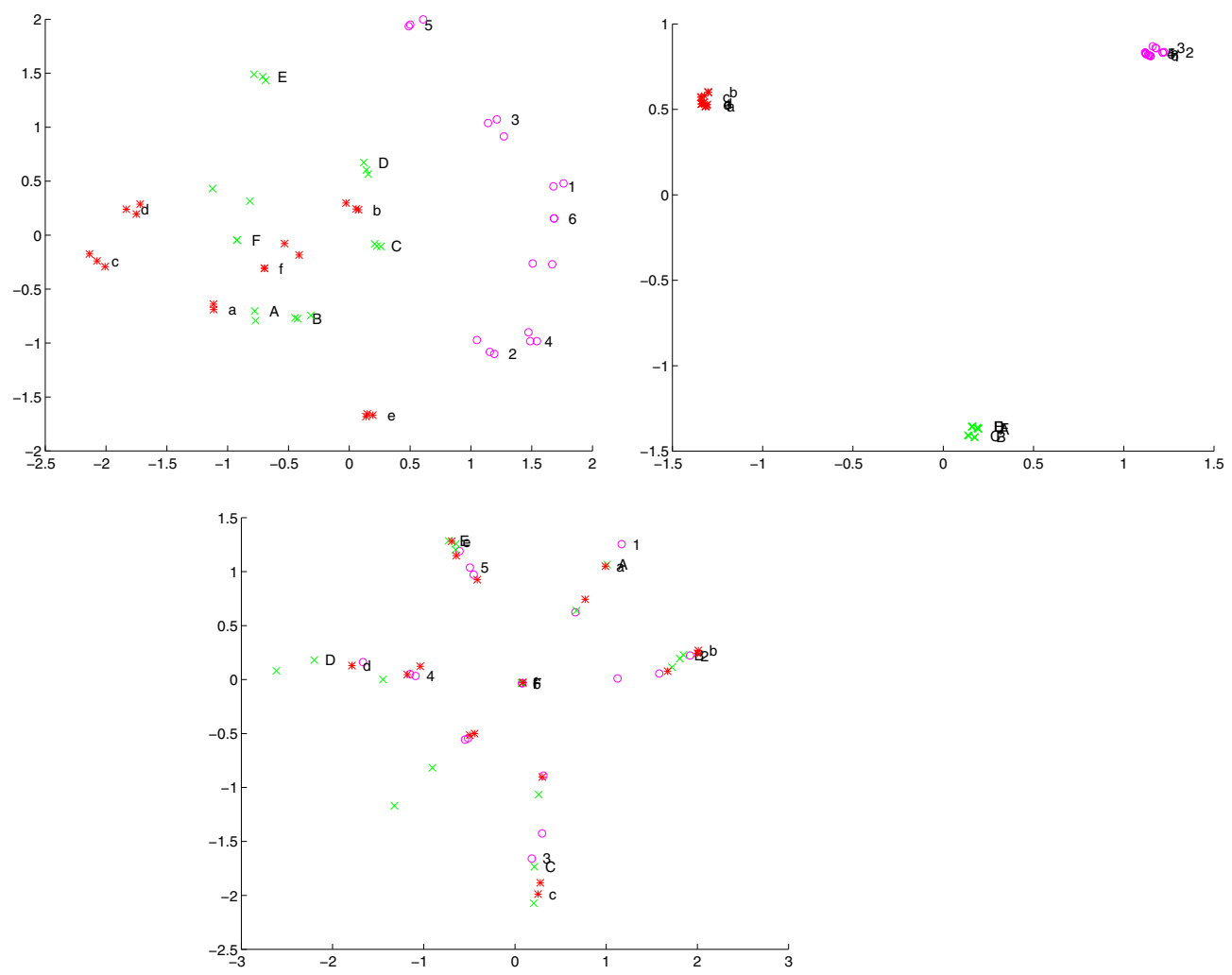

Figure 11. FRACTALS data, LDR by 1-HL MLP, MDS visualization. This figure illustrates the incorporation of prior hierarchical category knowledge into the LDR extraction process. Top left: LDR derived by a 1-HL MLP with 5 hidden units, trained to produce a unary encoding of the 18-way label set, to which a coarser set of 3-way class labels, corresponding to the row number in figure 1 , has been appended (also in a unary format). The row variables were given a weight of $w_{c}=0.1$ relative to the identity variables. The test dichotomy error rate on the resulting LDR was 0.056. Top right: results for an MLP trained to produce a unary encoding of the 3 row and the 6 column numbers of the stimulus. The test dichotomy error rate on the resulting LDR was 0.056. The relative weights of the row and the column variables were $w_{r}=1.0$, $w_{c}=0.75$. Bottom: same $3 \times 6$ class structure as before, but the relative weights of the row and the column variables were $w_{r}=0.05, w_{c}=1.0$. The test dichotomy error rate on the resulting LDR was 0.20 . The test dichotomy error rate on the raw data in all three cases was 0.28 .

corresponding to the rows and six labels corresponding to the columns of the parameterspace configuration. The resulting configuration depended to a significant degree on the relative weights given to the row and column labels. Under nearly equal weights, the points were separated into three clusters by the row label (see figure 11, top right); when the column weight predominated, the separation was into six clusters (i.e. by column), with some additional structure within each cluster (see figure 11, bottom).

A natural extrapolation of this strategy would be to teach the network many possible dichotomies, in the hope that the structure of the underlying LDR can be recovered from the multiple two-way classifications (Price et al 1995). The advantage of operating at the level of 18 classes (or of three classes, with six subclasses each) is in the much shorter training procedure. On the other hand, training on multiple dichotomies may have the advantage of forcing the LDR extractor to consider multiple, hopefully disjoint, sets of 

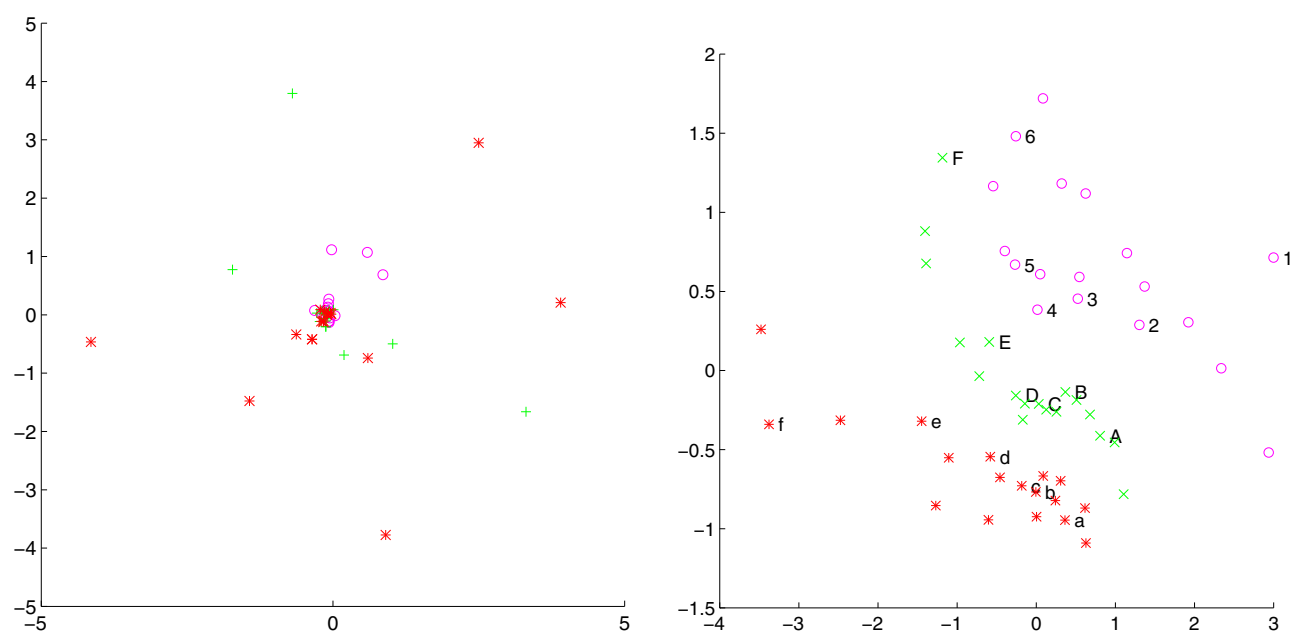

Figure 12. Left: FRACTALS data; the pattern recovered by MDS from the raw 784-dimensional data set. No separation of the 18 classes is apparent; most of the points are concentrated in the middle of the plot (cf figure 7, left). Right: FACES data; the pattern recovered by MDS from the raw 784-dimensional data set. Although the general layout of the 18 classes relative to one another is preserved, the points belonging to different classes are not separated (cf figure 4, left). Indeed, MDS by itself had no reason to separate the classes, unlike the MLP, which has been explicitly trained to do so (but not necessarily to preserve the 2D metric layout of the classes relative to each other, which it did, just like MDS!).

features relevant to the collection of tasks, and not letting it zero in on distinctive features specific for each one-versus-all discrimination. We leave for future research the quest for an optimal compromise between these considerations.

\subsection{The FACES data set}

LDR extraction from the FACES data set was easier than from the FRACTALS, as could be expected from the comparison of the degree of nonlinearity of the two sets, described in section 2. This expectation was supported by another comparison-between the configurations derived by MDS from the raw 784-dimensional FRACTALS and FACES data (see figure 12) - and was confirmed by the results of the experiments involving the FACES data set, which we describe next.

Good results were obtained on the FACES data using all three methods for LDR extraction: 1-HL MLP, 3-HL bottleneck MLP and RBF. The performance of the MLPbased methods in recovering the topology of the row/column parametric structure of the 18 classes seems to be especially amazing (compare e.g. figure 13 (left) with the labels in figure 5 (top); see also figures 14-16). Importantly, this recovery was possible even when the network was trained on half of the 18 classes, then tested on the full data set (see figure 16). The implications of this and the other results are discussed in section 6 .

\section{Control experiments}

The difficulty of LDR extraction in the present case is demonstrated by a comparison to the results obtained by more conventional neural network methods for dimensionality 

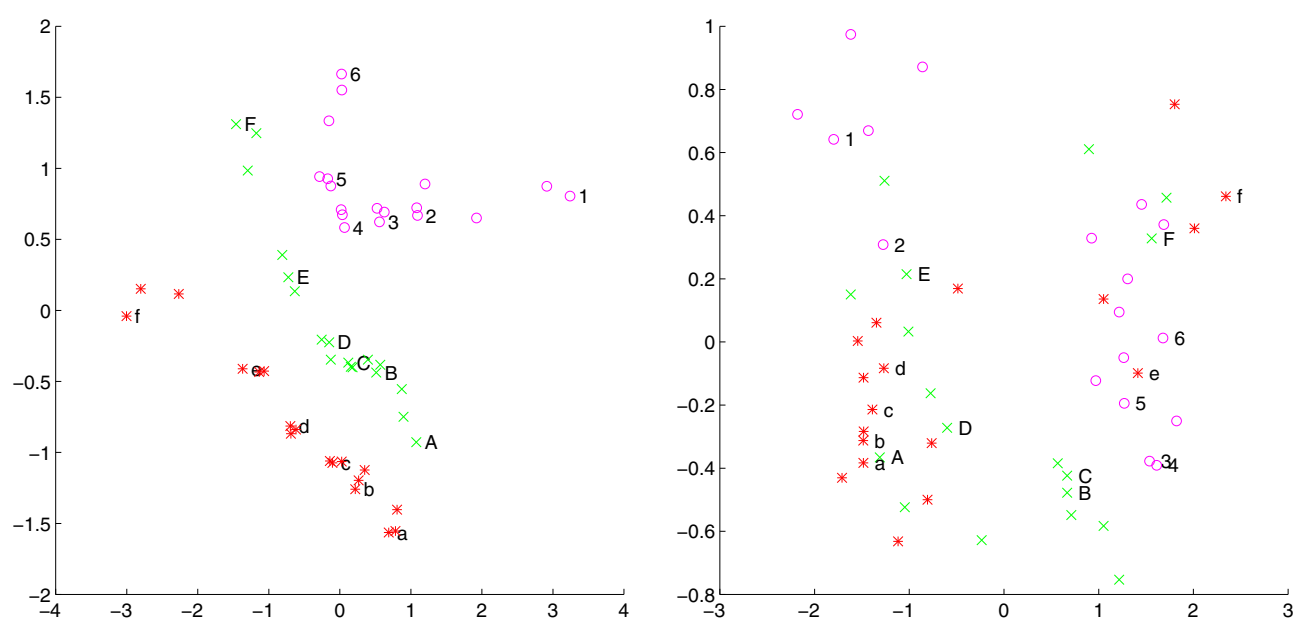

Figure 13. FACES data, MDS visualization (section 3.2.2). Left: LDR by 1-HL MLP with 13 hidden units (section 3.1.1), trained for 20000 epochs on the 18-class task. The test dichotomy error rate was 0.02 , compared to 0.07 on the raw data. Right: control results for a 1-HL MLP with 13 hidden units, trained for 20000 epochs on a dichotomy task. The test dichotomy error rate was 0.04 , compared to 0.07 on the raw data.
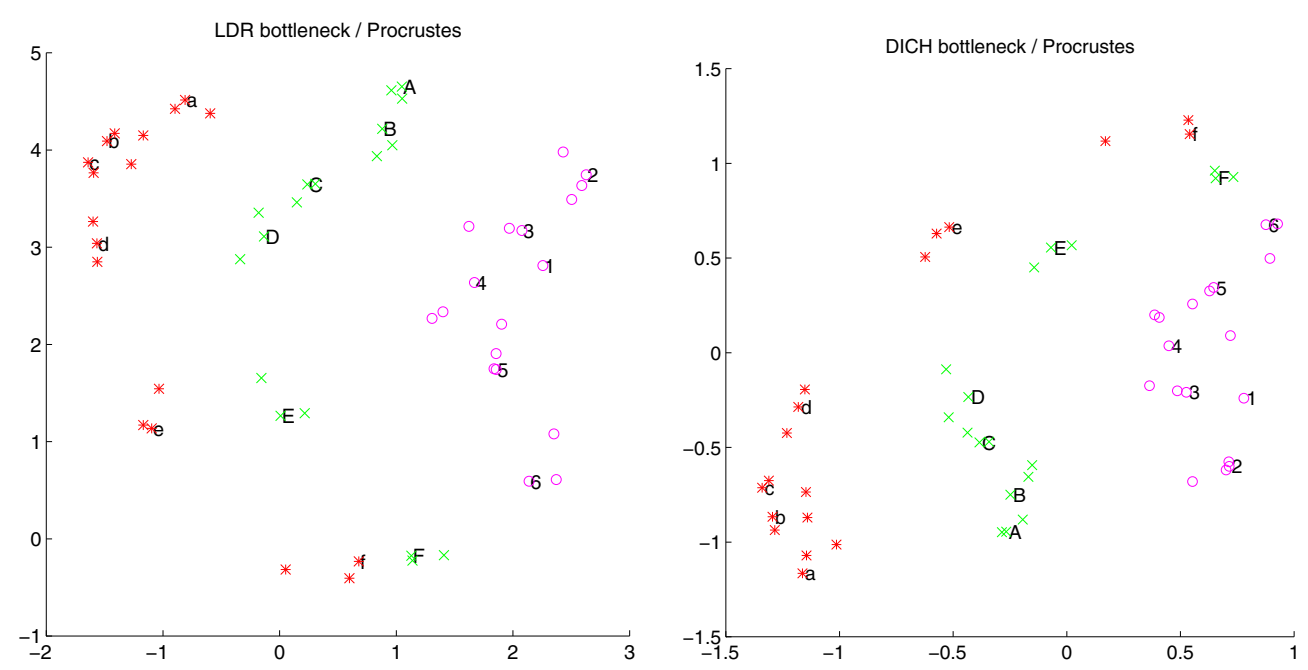

Figure 14. FACES data, LDR by $3-\mathrm{HL}$ bottleneck MLP, MDS visualization. Left: results for 3-HL bottleneck MLP with two hidden units, trained on the 18-class task. The test dichotomy error rate was 0.1 , compared to 0.29 on the raw data. Right: results for 3-HL bottleneck MLP, trained as an autoencoder on the LDR derived from the middle HL of the previous 3-HL MLP.

reduction. The best-known such methods employ self-supervised bottleneck autoencoder training. While the imposition of a low-dimensional bottleneck is common to these methods and to our approach, there is a crucial difference: an autoencoder is trained to reproduce the data while our networks are trained to assign the data a certain category structure. To characterize the importance of this feature of our approach to the extraction of useful LDRs, we conducted several experiments. 

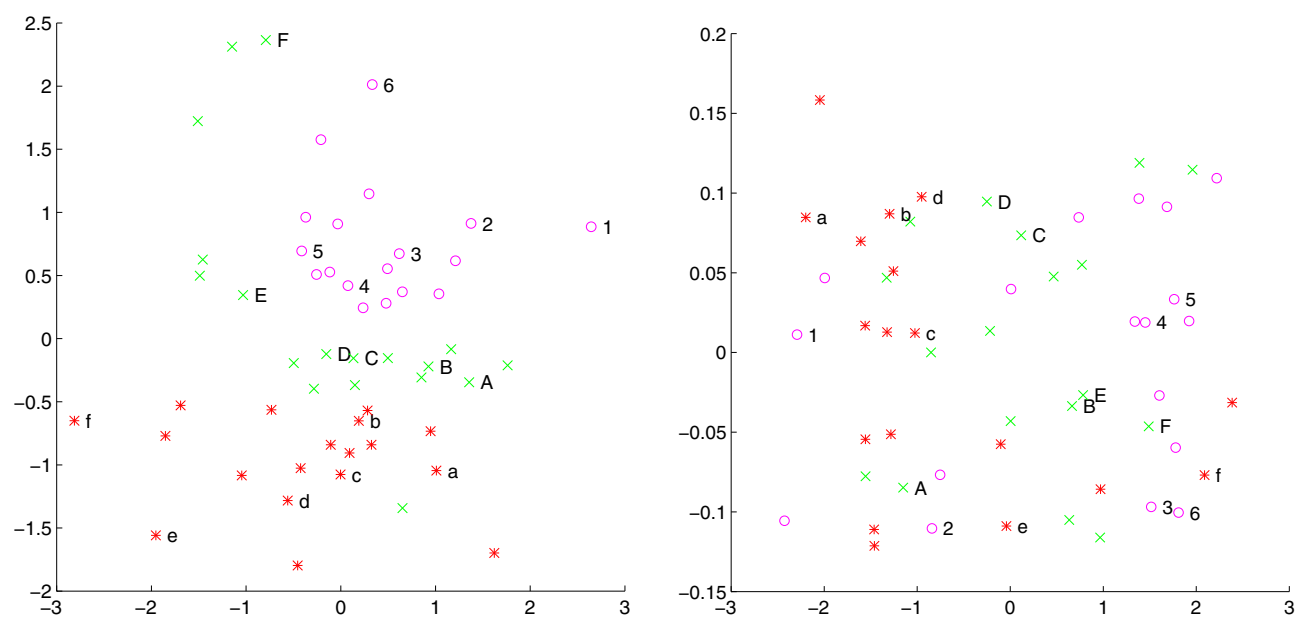

Figure 15. FACES data, MDS visualization. Left: an 18-centre RBF (section 3.1.3), trained on the 18 -class task. The test dichotomy error rate was 0.04 , compared to 0.07 on the raw data Recall that each point corresponds to one test view; the three views belonging to each of the 18 test faces are usually grouped together. The labels should be compared with those in figure 4 . Right: an 18-centre RBF, trained on the dichotomy. The test dichotomy error rate was 0.11 , worse than the error rate of 0.07 on the raw data.

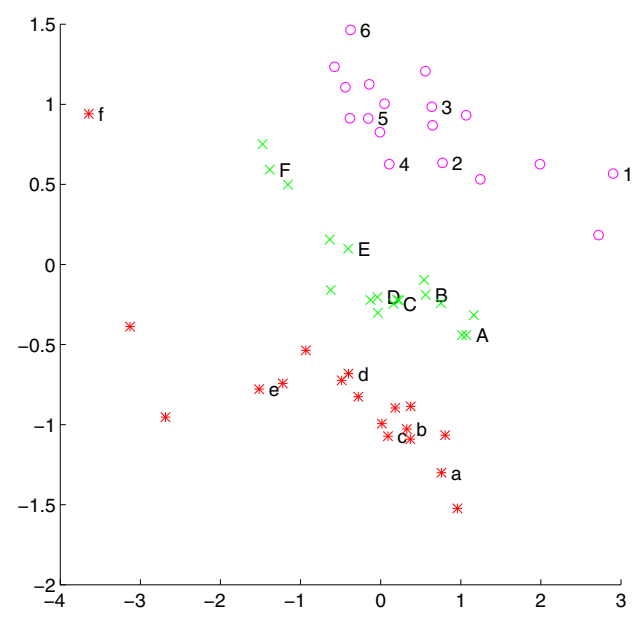

Figure 16. FACES data set, LDR by a 1-HL multilayer perceptron (MLP), trained on every second class; MDS visualization. These results were obtained with a 1HL MLP network with nine hidden units, trained on half of the 18 classes that comprise the problem space (the nine classes used for training and the nine omitted classes formed a checkerboard pattern). Note that all 18 classes-both familiar ones and those not seen by the system-are in a topology-preserving formation. The test dichotomy error rate was 0.14 , compared to 0.28 on the raw data.

First, we asked whether a self-supervised three-layer MLP autoencoder, which aims at the best reconstruction of the inputs, can reveal the correct low-dimensional structure in our data. Although in the linear case such networks do quite well, essentially by extracting the principal components of the data (Elman and Zipser 1988), the performance on the FACES data was poor. Specifically, the autoencoder network consistently converged to the mean of the data, presumably due to the nonlinearity introduced by the imaging step.

Second, we experimented with a five-layer nonlinear bottleneck autoencoder (Leen and Kambhatla 1994). This training scheme, likewise, performed poorly on our data set. The outcome of this experiment showed that self-supervised dimensionality reduction cannot 
recover a good LDR in the present case, illustrating the importance of guidance provided by the class labels.

A bottleneck autoencoder employed as a dimensionality reducing device is required to map a high-dimensional space (in our case, a space of 784 dimensions) to itself, whereas the output space in our training scheme was 18-dimensional. One may ask here whether a certain reduction in the dimensionality of the output space, combined with an imposition of certain category structure on that space, would enable the network to learn the proper LDR. To address this question, we tested a modified version of our method, in which the classifier was not trained to ignore the direction orthogonal to the target manifold (cf figure 17; this was done by training on the 72 face-view labels, instead of the 18 face identity labels). Thus, the network's output space was 72-dimensional, with each dimension corresponding to a conjunction of face identity and face orientation labels. This manipulation, however, did not help: the LDR extracted by the modified autoencoder was poor, underscoring the importance of guidance provided by an explicit specification of the dimension to be collapsed (in this case, the dimension of the viewpoint-related variation).

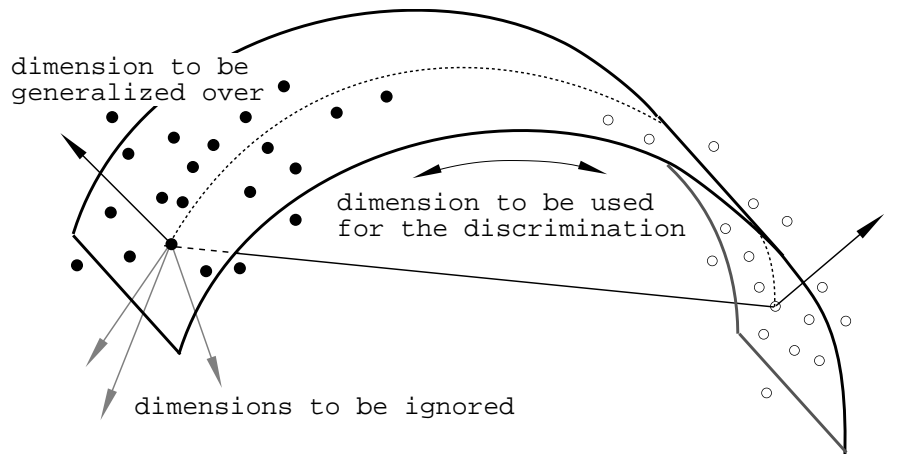

Figure 17. A schematic illustration of a problem space whose efficient representation requires nonlinear dimensionality reduction. The instances of the two classes cling to a low-dimensional manifold, embedded in a measurement space, whose dimensionality may run in the tens of thousands. See section 6 for a discussion of this example.

\section{Conclusions}

We have shown that combining multiple constraints via the use of multiple-class labels is an effective way to impose bias on a learning system whose goal is to find a good LDR. In particular, the use of multiple-class labels steers the system to become invariant to those directions of variation in the input space that play no role in the classification tasks. This is done merely by using class labels that are independent of these directions: in the Fractal images, the image labels are invariant to the third dimension of variation (figure 1, left); similarly, the identity labels of the FACES are independent of their orientation (figure 5, bottom panel). We have also shown that prior knowledge of the 'proper' dimensionality of the target LDR can be imposed by training a multi-layer bottleneck network (figure 6, bottom right). Both these features of our approach lead to improved generalization in the learned tasks.

A useful intuition concerning the effectiveness of our method can be developed by considering an analogy with discriminant analysis, a well-known technique for projecting data onto dimensions important for a particular classification task (see figure 17). Assume 
that some dimensions of a set of measurements performed on the world are crucial for distinguishing between the categories, while other dimensions must be downplayed, or collapsed; in the context of object recognition, the former may be the dimensions of object identity, and the latter those of object orientation. Whereas standard discriminant analysis methods in multidimensional spaces are plagued by the presence of irrelevant dimensions, in this paper we have shown that training with a combined objective of (1) discrimination among labelled categories known to reside within the manifold, and (2) explicit collapse of dimensions over which discrimination is to be generalized, leads to an improved performance in other discrimination tasks involving the same objects, and to a reliable recovery of a lowdimensional manifold containing the objects, even when it is curved (i.e. when the problem is nonlinear) and is embedded in a measurement space of nearly a thousand dimensions. This approach can be compared with a recent suggestion to convert poor features into supervisors (Caruana and de Sa 1997); in contrast to that idea, we show that there is a limit to the utility of class labels, and that invariance constraints we impose are equally important.

An important feature of the LDR computed by our method is the preservation of the topology of the parameter space underlying the data. The clusters corresponding to the labels we imposed during training were both separated from each other (as dictated by the training procedure), and arranged in the resulting low-dimensional space in a pattern that reflected their arrangement in the parameter space used to create the data. This latter property of the representation was obtained even when the network had been trained only on half of the objects over which topology preservation was evaluated.

Topology preservation is useful because it allows the representational system to categorize novel instances of familiar object classes, as well as make sense of novel classes (Edelman and Duvdevani-Bar 1997b). Specifically, if proximities in the representation space reflect similarities among objects 'out there' in the world, the following two operations can be carried out safely: (1) a new instance of a class can be categorized by finding, in the representation space, the cluster to which the current stimulus is the closest; (2) a new class can be defined by its representation-space distances to the familiar classes (Edelman and Duvdevani-Bar 1997a) $\dagger$.

We remark that topology preservation appears to be true of representation formed by human subjects in a variety of perceptual tasks. Studies in experimental psychology indicate that a low-dimensional pattern built into complex 2D shapes (by arranging these shapes in a conspicuous configuration in an underlying parameter space) is recovered by the visual system of subjects required to judge similarities between the shapes (Shepard and Cermak 1973, Cortese and Dyre 1996). Recently, similar findings have been achieved in experiments that involved 3D objects, arranged in a variety of planar configurations in a parameter space of several dozen dimensions (Edelman 1995a, Cutzu and Edelman 1996). The upshot of these findings is that the human visual system is capable of recovering the proper lowdimensional representation of the stimuli from a million-dimensional measurement space (dictated by the number of axons leading from the retina to the brain), while preserving the topology of the original space (and in many cases the exact relative placement of the stimuli in that space). The conditions on the LDR extraction process that makes such recovery possible, and the wider philosophical implications of this phenomenon, are discussed in (Edelman 1997).

$\dagger$ In the context of figure 16, this operation corresponds to the definition of a novel face (say, face B, which was not included in the training set) in terms of its similarities to familiar faces (e.g. defining B as the stimulus that is halfway between A and C, as well as between 2 and b). 


\section{Acknowledgments}

We thank P Dayan and J Tenenbaum for useful suggestions.

\section{Appendix: Multidimensional scaling (MDS)}

MDS was originally developed in psychometrics, as a method for the recovery of the coordinates of a set of points from measurements of the pairwise distances between those points (Young and Householder 1938). In a typical application, the experimenter would attempt to characterize a subject's performance by placing a point corresponding to each stimulus perceived by the subject in a coordinate space, derived from subjective similarity ratings of pairs of stimuli. The power of MDS as a tool for the study of internal representations (of human subjects) was revealed when Shepard discovered in 1962 that fixing the relative distances of a set of points effectively determines their coordinates (Shepard 1966). This discovery led to the development of the non-metric MDS algorithm (Kruskal 1964), which employs gradient descent to seek a monotonic transformation between measured distances and distances computed from the hypothesized point configuration, which would minimize stress (defined as the discrepancy between the ranks of the measured and the computed distances). In the present work, we used a modern implementation of nonmetric MDS, available in version 6 of the SAS statistical analysis software (SAS 1989), to allow the visualization of high-dimensional data sets. The points in a given set were embedded into a 2D metric space reflecting as closely as possible the pattern of inter-point distances, then plotted and subjected to inspection.

\section{References}

Atick J J, Griffin P A and Redlich A N 1996 The vocabulary of shape: principal shapes for probing perception and neural response Network: Comput. Neural Syst. 7 1-5

Baldi P and Hornik K 1989 Neural networks and principal component analysis: Learning from examples without local minima Neural Networks 2 53-8

Baxt W G and White H 1995 Bootstrapping confidence intervals for clinical input variable effects in network trained to identify the presence of acute myocardial infraction Neural Comput. 7 624-38

Bellman R E 1961 Adaptive Control Processes (Princeton, NJ: Princeton University Press)

Borg I and Lingoes J 1987 Multidimensional Similarity Structure Analysis (Berlin: Springer)

Breiman L 1992 Stacked regression Technical Report TR-367 Department of Statistics, University of California, Berkeley

Breiman L 1994 Bagging predictors Technical Report TR-421 Department of Statistics, University of California, Berkeley

Buckheit J and Donoho D L 1995 Improved linear discrimination using time-frequency dictionaries Technical Report Stanford University

Busey T A, Brady N P, and Cutting J E 1990 Compensation is unnecessary for the perception of faces in slanted pictures Perception Psychophys. 48 1-11

Caruana R 1993 Multitask connectionist learning Proc. 1993 Connectionist Models Summer School (San Mateo, CA: Morgan Kaufmann) pp 372-9

Caruana R 1995 Learning many related tasks at the same time with backpropagation Advances in Neural Information Processing Systems 7 ed G Tesauro, D Touretzky and T Leen pp 657-64 (San Mateo, CA: Morgan Kaufmann) pp 657-64

Caruana R and de Sa V R 1997 Promoting poor features to supervisors: Some inputs work better as outputs Advances in Neural Information Processing Systems 9 ed M C Mozer, M I Jordan and T Petsche (San Mateo, CA: Morgan Kaufmann)

Cortese J M and Dyre B P 1996 Perceptual similarity of shapes generated from Fourier Descriptors J. Exp. Psychol: Human Percept. Perform. 22 133-43 
Cottrell G W, Munro P and Zipser D 1987 Learning internal representations from gray-scale images: An example of extensional programming Proc. 9th Ann. Conf.of the Cognitive Science Society (Hillsdale, NJ: Erlbaum) pp 462-73

Cutzu F and Edelman S 1996 Faithful representation of similarities among three-dimensional shapes in human vision Proc. Natl Acad. Sci. USA 93 12046-50

Demartines P and Hérault J 1996 Curvilinear component analysis: a self-organizing neural network for non linear mapping of data sets IEEE Trans. Neural Networks submitted

DeMers D and Cottrell G 1993 Nonlinear dimensionality reduction Advances in Neural Information Processing Systems 5 ed S J Hanson, J D Cowan and C L Giles (San Mateo, CA: Morgan Kaufmann) pp 580-7

Edelman S 1995a Representation of similarity in 3D object discrimination Neural Comput. 7 407-22

- 1995b Representation, similarity, and the chorus of prototypes Minds Machines 5 45-68

1997 Representation is representation of similarity Behav. Brain Sci. in press

Edelman S, Cutzu F and Duvdevani-Bar S 1996 Similarity to reference shapes as a basis for shape representation Proc. 18th Ann. Conf. of Cognitive Science Society (San Diego, CA) ed G W Cottrell (Hillsdale, NJ: Erlbaum) pp 260-5

Edelman S and Duvdevani-Bar S 1997a A model of visual recognition and categorization Phil. Trans. R. Soc. Lond. B 352 in press

-1997b Similarity, connectionism, and the problem of representation in vision Neural Comput. 9 701-20

Efron B and Tibshirani R 1993 An Introduction to the Bootstrap (London: Chapman and Hall)

Elman J L and Zipser D 1988 Learning the hidden structure of speech J. Acoust. Soc. Am. 4 1615-26

Fisher R A 1936 The use of multiple measurements in taxonomic problems Ann. Eugenics 7 179-88

Grossman T and Lapedes A 1993 Use of bad training data for better prediction Advances in Neural Information Processing Systems 6 ed J D Cowan, G Tesauro and J Alspector (San Mateo, CA: Morgan Kaufmann) pp $342-50$

Intrator N 1993 Combining exploratory projection pursuit and projection pursuit regression with application to neural networks Neural Comput. 5 443-55

Intrator N and Edelman S 1996 How to make a low-dimensional representation suitable for diverse tasks Connect. Sci. 8 205-24

Koontz W L G and Fukunaga K 1972 A nonlinear feature extraction algorithm using distance information IEEE Trans. Comput. C-21 56-63

Kruskal J B 1964 Multidimensional scaling by optimizing goodness of fit to a nonmetric hypothesis Psychometrika 29 1-27

Lando M and Edelman S 1995 Receptive field spaces and class-based generalization from a single view in face recognition Network: Comput. Neural Syst. 6 551-76

LeBlanc M and Tibshirani R 1994 Combining estimates in regression and classification Preprint Stanford University

Leen T K and Kambhatla N 1994 Fast non-linear dimension reduction Advances in Neural Information Processing Systems 6 ed J D Cowan, G Tesauro and J Alspector (San Mateo, CA: Morgan Kaufmann) pp 152-9

Lowe D 1993 Novel 'topographic' nonlinear feature extraction using radial basis functions for concentration codeing in the 'artificial nose' Proc. 3rd IEE Int. Conf. on Artificial Neural Networks (Stevenage: IEE)

Lowe D and Tipping M E 1996 Feed-forward neural networks and topographic mappings for exploratory data analysis Neural Computing Appl. 4 83-95

Nowlan S J and Hinton G E 1992 Simplifying neural networks by soft weight-sharing Neural Comput. 4 473-93

Oja E 1989 Neural networks, principal components, and subspaces Int. J. Neural Syst. 1 61-8

Pickover C 1990 Computers, Pattern, Chaos, and Beauty (New York: St. Martin's Press)

Poggio T and Girosi F 1990 Regularization algorithms for learning that are equivalent to multilayer networks Science 247 978-82

Price D, Knerr S, Personnaz L and Dreyfus G 1995 Pairwise neural network classifiers with probabilistic outputs Advances in Neural Information Processing 7 ed G Tesauro, D S Touretsky and T K Leen (Cambridge, MA: MIT Press) pp 1109-16

Raviv Y and Intrator N 1996 Bootstrapping with noise: An effective regularization technique Connect. Sci. 8 356-72

Sammon J W 1969 A nonlinear mapping for data structure analysis IEEE Trans. Comput. C-18 401-9

SAS 1989 SAS/STAT User's Guide, Version 6 SAS Institute Inc., Cary, NC

Shepard R N 1966 Metric structures in ordinal data J. Math. Psychol. 3 287-15

Shepard R N 1980 Multidimensional scaling, tree-fitting, and clustering Science 210 390-7

Shepard R N and Cermak G W 1973 Perceptual-cognitive explorations of a toroidal set of free-form stimuli Cogn. Psychol. 4 351-77 
Siedlecki W, Siedlecka K and Sklansky J 1988 An overview of mapping techniques for exploratory pattern analysis Pattern Recogn. 21 411-29

Simard P, Victorri B, LeCun Y and Denker J 1992 Tangent prop-a formalism for specifying selected invariances in an adaptive network Neural Information Processing Systems 4 ed J Moody, R Lippman and S J Hanson (San Mateo, CA: Morgan Kaufmann) pp 895-903

Stone M 1974 Cross-validatory choice and assessment of statistical predictions (with discussion) J. R. Stat. Soc. B 36 111-47

Thrun S and Mitchell T 1995 Learning one more thing Proc. 14th Int. Joint Conf. on Artificial Intelligence, vol 2 ed C Mellish (San Mateo, CA: Morgan Kaufmann) pp 1217-23

Wahba G 1990 Splines Models for Observational Data (Series in Applied Mathematics 59) (Philadelphia, PA: SIAM)

Webb A R 1995 Multidimensional-scaling by iterative majorization using radial basis functions Pattern Recogn. 28 753-9

Young G and Householder A S 1938 Discussion of a set of points in terms of their mutual distances Psychometrika 3 19-22 